\title{
Scalar tachyons in the de Sitter universe
}

\author{
Jacques Bros ${ }^{1}$, Henri Epstein ${ }^{2}$ and Ugo Moschella ${ }^{3}$ \\ ${ }^{1}$ Service de Physique théorique - CEA. Saclay. 91191 Gif-sur Yvette. \\ ${ }^{2}$ Institut des Hautes Études Scientifiques, 91440 Bures-sur-Yvette. \\ ${ }^{3}$ Università dell'Insubria, Como and INFN Milano
}

May 2, 2019

\begin{abstract}
We provide a construction of a class of local and de Sitter covariant tachyonic quantum fields which exist for discrete negative values of the squared mass parameter and which have no Minkowskian counterpart. These quantum fields satisfy an anomalous non-homogeneous Klein-Gordon equation. The anomaly is a covariant field which can be used to select the physical subspace (of finite codimension) where the homogeneous tachyonic field equation holds in the usual form. We show that the model is local and de Sitter invariant on the physical space. Our construction also sheds new light on the massless minimally coupled field, which is a special instance of it.
\end{abstract}

The word tachyon denotes a would-be particle traveling faster than light. Despite the fact that tachyons are believed to be unphysical, they play an important role in many circumstances as for instance in bosonic string theory, and the question about their existence and physical meaning is far from being settled. The commonly accepted interpretation is that the appearance of tachyonic degrees of freedom in a theoretical model points towards an instability of the model, and this instability should be described by tachyonic fields as opposed to real faster-than-light particles; the instability can be treated by invoking the so-called tachyon condensation.

Starting with the observation of distant supernovae in 1998, the cosmological constant has taken a central place in the scene of contemporary physics; since then many widespread beliefs have been shattered and abandoned. A nonzero cosmological constant may also be responsible for a change in the understanding we have of tachyons, as we do show in this letter. A nonzero cosmological constant renders indeed possible the existence of a class of tachyonic fields on the de Sitter universe that do not share the problems of their corresponding Minkowskian counterparts, and on the contrary give rise to acceptable free field theories.

We will discuss the $d$-dimensional de Sitter manifold with unit radius, described as the hyperboloid $X_{d}=\left\{x \in \mathbf{R}^{d+1}, x^{2}=-1\right\}$. On this manifold we consider Klein-Gordon fields for general complex values of the squared mass $m^{2}=-\lambda(\lambda+d-1)$; tachyons correspond to real negative squared masses. Since we are dealing with free fields, the knowledge of a two-point function solving the de Sitter KleinGordon equation

$$
\square w_{\lambda}\left(x_{1}, x_{2}\right)=\lambda(\lambda+d-1) w_{\lambda}\left(x_{1}, x_{2}\right)
$$

w.r.t. both $x_{1}$ and $x_{2}$ allows for a complete reconstruction of the field. One would like to impose the following properties on the two-point function:

- invariance under the global symmetry group: for $X_{d}$, the latter is the de Sitter group $S O_{0}(1, d)$;

- locality, i.e. the vanishing of the commutator

$$
c_{\lambda}\left(x_{1}, x_{2}\right)=w_{\lambda}\left(x_{1}, x_{2}\right)-w_{\lambda}\left(x_{2}, x_{1}\right)
$$


at spacelike separation and

- positive-definiteness, to warrant a bona-fide quantum mechanical interpretation.

The above requirements have proven to be incompatible for tachyonic fields on the Minkowski manifold. Feinberg [1] considers tachyons as scalar fermions abandoning the locality condition while Schroer 2] constructs local and covariant tachyon fields which do not satisfy the positive-definiteness condition and have no direct quantum mechanical interpretation.

Even when the squared mass is positive, the above properties do not uniquely prescribe the two-point function and one needs physical or mathematical criteria to discriminate among the various possibilities. The "preferred vacua" of free de Sitter QFT's, also known as the Bunch-Davies vacua or "Euclidean vacua" [4, 6, 5, 3, 7, 8, can be uniquely identified by the property of maximal analyticity of their twopoint functions [9, 10, i.e. the latter arise as boundary values of functions which are analytic in the cut domain

$$
\Delta=\left\{\left(z_{1}, z_{2}\right) \in X_{d}^{(c)} \times X_{d}^{(c)}:\left(z_{1}-z_{2}\right)^{2}=-2-2 z_{1} \cdot z_{2} \neq c>0\right\}
$$

$X_{d}^{(c)}$ being the complex de Sitter hyperboloid $X_{d}^{(c)}=\left\{z \in \mathbf{C}^{d+1}, z^{2}=-1\right\}$; the cut is the projection on the complex plane of the invariant variable $\left(z_{1}-z_{2}\right)^{2}$ of pairs of real points $\left(z_{1}, z_{2}\right)$ that have timelike or lightlike separation. The analyticity of the two-point function in $\Delta$ therefore encodes the quantum mechanical requirement that field operators at spacelike separations commute, while those at timelike separations, in general, do not. The maximal analyticity property has been shown to be equivalent to a certain thermal KMS property [10. It is the closest analog of the analyticity property implied by the positive energy condition for the case of Minkowskian field theories. Other vacua (e.g. the so called alpha-vacua [8]) do not share these analyticity properties and have no temperature.

For each (real or complex) value of the mass parameter $m$ (or $\lambda$ ), the relevant two-point function, solution of Eq (0.1), is uniquely determined by the above analyticity properties, invariance, and the CCR; it can be expressed in the complex domain in terms of the hypergeometric function as follows

$$
\begin{aligned}
& w_{\lambda}\left(z_{1}, z_{2}\right)=\Gamma(-\lambda) G_{\lambda}(\zeta), \quad \zeta=z_{1} \cdot z_{2} \\
& G_{\lambda}(\zeta)=\frac{\Gamma(\lambda+d-1)}{(4 \pi)^{d / 2} \Gamma\left(\frac{d}{2}\right)} F\left(-\lambda, \lambda+d-1 ; \frac{d}{2} ; \frac{1-\zeta}{2}\right) .
\end{aligned}
$$

These two-point functions are closely connected to the irreducible representations of the de Sitter group and their spherical functions, studied in a number of mathematical works, see e.g. [12, 14, 16, 15, 13, 11] and references therein.

- Theories in the above preferred family corresponding to $m^{2}>0$ (and the limiting massless minimally coupled case $m^{2}=0$ ) have been widely studied in the literature with extremely important applications to inflation, and their physical interpretation is still under active discussion [17, 18, 19, 20,

- Theories corresponding to negative or, more generally, complex squared masses give rise to quantum fields which are local and de Sitter invariant but do not satisfy the positive-definiteness condition; one can reconstruct a linear space of states but the inner product naturally associated with those two-point functions does not yield a positive norm, and therefore does not give rise to a Hilbert space; nor can a de Sitter invariant positive subspace be found in the above linear space. Therefore there is no acceptable quantum interpretation for the field theories in this family.

There is however an exceptional family of masses $m^{2}=-n(n+d-1)$ corresponding to nonnegative integer $\lambda=n$ (in the $n=0$ case, we get the massless minimally coupled field; see 21 for a precursor of this paper), for which the two-point function is simply infinite because of the pole of $\Gamma(-\lambda)$ at $\lambda=n$ in the RHS of Eq. (0.4); recall that the normalization of $w_{\lambda}$ in (0.4) is determined by imposing the 
canonical commutation relations. On the other hand

$$
F\left(-n, n+d-1 ; \frac{d}{2} ; \frac{1-\zeta}{2}\right)=\frac{\Gamma(n+1) \Gamma(d-1)}{\Gamma(n+d-1)} C_{n}^{\frac{d-1}{2}}(\zeta)
$$

reduces to a Gegenbauer polynomial of degree $n$ (see [22]); it is a holomorphic function without cuts and consequently with no quantum feature.

We may try to give a meaning to this family of theories by subtracting the divergent part. Since the pole of $\Gamma(-\lambda)$ is simple, with residue $(-1)^{n+1} / n$ !, it is clear that the following two-point function is well-defined:

$$
\widehat{w}_{n}\left(z_{1}, z_{2}\right)=\widehat{w}_{n}(\zeta)=\lim _{\lambda \rightarrow n} \Gamma(-\lambda)\left[G_{\lambda}(\zeta)-G_{n}(\zeta)\right]=\left.\frac{(-1)^{n+1}}{n !} \frac{\partial}{\partial \lambda} G_{\lambda}(\zeta)\right|_{\lambda=n}
$$

Because $G_{n}(\zeta)$ has no discontinuity, the commutator $c_{\lambda}$ associated to $w_{\lambda}$ (see (0.2)) tends to a welldefined limit as $\lambda \rightarrow n$, without needing any subtraction, and this limit is precisely the commutator associated to $\widehat{w}_{n}$ :

$$
c_{n}(x, y)=\lim _{\lambda \rightarrow n} c_{\lambda}(x, y)=\widehat{w}_{n}(x, y)-\widehat{w}_{n}(y, x)
$$

While the commutator solves the true Klein-Gordon equation, the two-point function $\widehat{w}_{n}$ satisfies a modified tachyonic Klein-Gordon equation having an anomalous non-homogeneous RHS as follows:

$$
[\square-n(n+d-1)] \widehat{w}_{n}(\zeta)=\frac{(-1)^{n+1}(2 n+d-1) \Gamma\left(\frac{d-1}{2}\right)}{4 \pi^{\frac{d+1}{2}}} C_{n}^{\frac{d-1}{2}}(\zeta),
$$

In the massless minimally coupled case the above equation becomes simpler

$$
\square \widehat{w}_{0}(\zeta)=-\frac{\Gamma\left(\frac{d+1}{2}\right)}{2 \pi^{\frac{d+1}{2}}} ;
$$

the constant at the RHS is the inverse of the hypersurface of the unit sphere in dimension $d+1$. This is related to the fact that the Laplace operator on the Euclidean de Sitter sphere has a zero mode and that our subtraction scheme in this case amounts exactly to the removal of that zero mode. In the procedure (0.7) leading to $\widehat{w}_{n}$, any meromorphic function of $\lambda$ with a simple pole at $\lambda=n$ could have been used instead of $\Gamma(-\lambda)$ : the result would have differed from $\widehat{w}_{n}$ by the addition of a multiple of $C_{n}^{\frac{d-1}{2}}(\zeta)$, without affecting (0.9).

Preserving a local and de Sitter invariant quantization of this family of field theories (including the massless minimally coupled scalar) is thus possible at the expense of an anomalous non-homogeneous term in the quantum field equations:

$$
[\square-n(n+d-1)] \phi=Q_{n}
$$

Of course we are really interested in (tachyonic) fields satisfying the correct field equation with no non-homogeneous term. We are therefore tempted to impose the condition

$$
Q_{n}^{-} \Psi=0
$$

on physical states, where $Q_{n}^{-}$denotes the "annihilation" part of the operator $Q_{n}$. An extraordinary property is that the above condition also selects a positive and de Sitter invariant subspace of the space of local states and therefore opens the way for an acceptable quantum mechanical interpretation of the de Sitter tachyons (associated with the discrete series of representations of the de Sitter group). Full proofs will be published elsewhere. Here we only give a hint of the proof of this property in the one-particle subspace of the model. In this case the condition is

$$
\Psi \in \mathcal{E}_{n}, \quad \mathcal{E}_{n}=\left\{\Psi \in \mathcal{C}_{0}^{\infty}\left(X_{d}\right): \int C_{n}^{\frac{d-1}{2}}\left(x_{1} \cdot x_{2}\right) \Psi\left(x_{2}\right) d x_{2}=0\right\}
$$


where $d x$ is the de Sitter invariant measure on the hyperboloid $X_{d} . \mathcal{E}_{n}$ is manifestly a de Sitter invariant subspace of the one-particle local states. The positivity in this subspace is a consequence of the following Fourier-type (i.e. a "momentum space") representation of the two-point function $\widehat{w}_{n}\left(z_{1}, z_{2}\right)$ :

$$
\begin{gathered}
\widehat{w}_{n}\left(z_{1}, z_{2}\right)=\widehat{W}_{n}\left(z_{1}, z_{2}\right)-F_{n}^{1}\left(z_{1}, z_{2}\right)-F_{n}^{2}\left(z_{1}, z_{2}\right)+b_{n} G_{n}\left(z_{1}, z_{2}\right) . \\
\widehat{W}_{n}\left(z_{1}, z_{2}\right)=a_{n} \int_{\gamma} \int_{\gamma}\left(z_{1} \cdot \xi\right)^{1-d-n}\left(\xi \cdot \xi^{\prime}\right)^{n} \log \left(\xi \cdot \xi^{\prime}\right)\left(z_{2} \cdot \xi^{\prime}\right)^{1-d-n} d \mu(\xi) d \mu\left(\xi^{\prime}\right) \\
F_{n}^{1}\left(z_{1}, z_{2}\right)=a_{n} \int_{\gamma} \int_{\gamma} \log \left(z_{1} \cdot \xi\right)\left(z_{1} \cdot \xi\right)^{1-d-n}\left(\xi \cdot \xi^{\prime}\right)^{n}\left(z_{2} \cdot \xi^{\prime}\right)^{1-d-n} d \mu(\xi) d \mu\left(\xi^{\prime}\right) \\
F_{n}^{2}\left(z_{1}, z_{2}\right)=a_{n} \int_{\gamma} \int_{\gamma}\left(z_{1} \cdot \xi\right)^{1-d-n}\left(\xi \cdot \xi^{\prime}\right)^{n} \log \left(z_{2} \cdot \xi^{\prime}\right)\left(z_{2} \cdot \xi^{\prime}\right)^{1-d-n} d \mu(\xi) d \mu\left(\xi^{\prime}\right)
\end{gathered}
$$

with

$$
a_{n}=\frac{(-1)^{n+1} \Gamma(n+d-1)^{2}}{2^{n+2 d} \pi^{\frac{3 d-1}{2}} n ! \Gamma\left(n+\frac{d-1}{2}\right)} \quad b_{n}=\left.\frac{(-1)^{n+1}}{n !} \frac{d}{d \lambda} \log \frac{\Gamma(\lambda+d-1)^{2}}{2^{\lambda+2 d} \pi^{\frac{3 d-1}{2}} \Gamma\left(\lambda+\frac{d-1}{2}\right)}\right|_{\lambda=n}
$$

Here $\xi$ and $\xi^{\prime}$ are vectors of the forward lightcone $C^{+}=\left\{\xi \in \mathbf{R}^{d+1}, \xi^{2}=0, \xi^{0}>0\right\}$; the integrals are taken over the section $\gamma=C_{+} \cap\left\{\xi: \xi^{0}=1\right\}$ i.e. over the unit sphere in $\mathbf{R}^{d}$; the measure $d \mu$ is the standard invariant measure on the unit sphere.

Eq. (0.14) is valid for any pair of complex de Sitter events $\left(z_{1}, z_{2}\right)$ such that $z_{1}$ belongs to the past tube $\mathcal{T}_{-}$and $z_{2}$ to the future tube $\mathcal{T}_{+}$, defined as follows: $\mathcal{T}_{ \pm}=\left\{z \in X_{d}^{(c)}, \operatorname{Im} z \in V^{ \pm}\right\}$; this encodes precisely the analyticity property of the preferred vacua [10]. The two-point function $\widehat{w}_{n}\left(x_{1}, x_{2}\right)$ is the boundary value on the reals from these tubes.

Note that the anomalous RHS in Eq. (0.9) is due to the terms $F_{n}^{1}$ and $F_{n}^{2}$. The proof that the restriction of the two-point function to $\mathcal{E}_{n} \times \mathcal{E}_{n}$ is de Sitter invariant and positive-definite is based on the following property: if a test function $f$ belongs to $\mathcal{E}_{n}$ the following integral vanishes

$$
\int_{\gamma} d \mu(\xi) p(\xi) \int_{X_{d}}(\xi \cdot(x \pm i 0))^{1-n-d} f(x) d x=0
$$

for any polynomial $p$ of degree $\leq n$. This fact implies in particular that only the first term $\widehat{W}_{n}\left(z_{1}, z_{2}\right)$ has a non-vanishing restriction to $\mathcal{E}_{n} \times \mathcal{E}_{n}$ :

$$
\left.\widehat{w}_{n}\left(z_{1} \cdot z_{2}\right)\right|_{\mathcal{E}_{n} \times \mathcal{E}_{n}}=\left.\widehat{W}_{n}\left(z_{1}, z_{2}\right)\right|_{\mathcal{E}_{n} \times \mathcal{E}_{n}} .
$$

The proof of positive-definiteness starts from the fact that $\xi \cdot \xi^{\prime}=1-\vec{\xi} \cdot \overrightarrow{\xi^{\prime}}$ for pair of points $\xi$ and $\xi^{\prime}$ belonging to the spherical section $\gamma$ of the cone. By inserting into Eq. (0.15) the power series expansion

$$
(-1)^{n+1}\left(1-\vec{\xi} \cdot \vec{\xi}^{\prime}\right)^{n} \log \left(1-\vec{\xi} \cdot \vec{\xi}^{\prime}\right) / n !=\sum_{m=0}^{\infty} u_{n, m}\left(\vec{\xi} \cdot \vec{\xi}^{\prime}\right)^{m}
$$

in the variable $\left(\vec{\xi} \cdot \vec{\xi}^{\prime}\right)$, we get

$$
\begin{aligned}
& \left.\widehat{w}_{n}\left(z_{1} \cdot z_{2}\right)\right|_{\mathcal{E}_{n} \times \mathcal{E}_{n}}=\frac{\Gamma(n+d-1)^{2}}{2^{n+2 d} \pi^{\frac{3 d-1}{2}} \Gamma\left(n+\frac{d-1}{2}\right)} \times \\
& \times \sum_{m=n+1}^{\infty} u_{n, m} \int_{\gamma \times \gamma}\left(z_{1} \cdot \xi\right)^{-n-d+1}\left(\vec{\xi} \cdot \vec{\xi}^{\prime}\right)^{m}\left(z_{2} \cdot \xi^{\prime}\right)^{-n-d+1} d \mu(\xi) d \mu\left(\xi^{\prime}\right)
\end{aligned}
$$


since terms of degree $\leq n$ do not contribute in the physical space. For $m>n$ the coefficients $u_{n, m}$ are easily shown to be positive real numbers; since the kernels $\left(\vec{\xi} \cdot \vec{\xi}^{\prime}\right)^{m}$ in the above integrals are all positive-definite, the positivity of the two-point function restricted to the de Sitter invariant one-particle physical space $\mathcal{E}_{n}$ follows.

The Fock construction finally produces a local and de Sitter covariant quantization of the tachyonic fields corresponding to the squared masses $m^{2}=-n(n+d-1)$. These tachyons disappear in the flat limit. More precisely one can consider the flat limit of the unrestricted two-point function $\widehat{w}_{n}\left(x_{1} \cdot x_{2}\right)$ : the limit exists and coincides with Schroer's two-point function, but the de Sitter invariant positive subspace gets smaller and smaller (though infinite dimensional!) and disappears in the limit.

It is straightforward to obtain from our definition (0.7) an explicit expansion of $\widehat{w}_{n}$ in terms of $z=$ $-\left(z_{1}-z_{2}\right)^{2} / 4=(1+\zeta) / 2$. In the case when $d \geq 2$ is an even integer, this expansion has a simple structure:

$$
\widehat{w}_{n}(\zeta)=z^{1-\frac{d}{2}} A(z, n, d)-\log (z) B(z, n, d)+C(z, n, d),
$$

where $A, B, C$ are polynomials in $z$ :

$$
\begin{aligned}
A(z, n, d) & =\sum_{m=0}^{\frac{d}{2}-2} \frac{z^{m} \Gamma\left(\frac{d}{2}-1-m\right) \Gamma\left(n+\frac{d}{2}+m\right)}{(4 \pi)^{\frac{d}{2}} \Gamma\left(n+\frac{d}{2}-m\right) m !} \\
B(z, n, d) & =\frac{\Gamma\left(\frac{d-1}{2}\right)}{4 \pi^{\frac{d+1}{2}}} C_{n^{\frac{d-1}{2}}}(1-2 z) \\
C(z, n, d) & =\sum_{m=0}^{n} \frac{(-1)^{m} z^{m} \Gamma(n+d-1+m)}{(4 \pi)^{\frac{d}{2}} \Gamma(1+m) \Gamma\left(\frac{d}{2}+m\right) \Gamma(n-m+1)} \times \\
& \times\left[\psi(1+m)+\psi\left(\frac{d}{2}+m\right)-2 \psi(n+d-1+m)-\psi(1+n)\right] .
\end{aligned}
$$

The most singular term in (0.23) is $(4 \pi)^{-\frac{d}{2}} \Gamma\left(\frac{d}{2}-1\right) z^{1-\frac{d}{2}}$. This reflects the normalization of $w_{\lambda}$, which is chosen so as to satisfy the canonical commutation relations. For example in dimension $d=4$,

$$
\widehat{w}_{0}(\zeta)=\frac{1}{(4 \pi)^{2} z}-\frac{2}{(4 \pi)^{2}} \log (z)-\frac{4-2 \gamma}{(4 \pi)^{2}}
$$

This exhibits the local Hadamard behavior of this two-point function. Only the first two terms in (0.23) contribute to the commutator $c_{n}\left(x_{1}, x_{2}\right)$ :

$$
\begin{aligned}
c_{n}\left(x_{1}, x_{2}\right)= & \sum_{p=1}^{\frac{d}{2}-1} \frac{2^{2 p+1} i \pi \Gamma(n+d-1-p) \epsilon\left(x_{1}^{0}-x_{2}^{0}\right) \delta^{(p-1)}\left(\left(x_{1}-x_{2}\right)^{2}\right)}{(4 \pi)^{d / 2} \Gamma(n+1+p) \Gamma\left(\frac{d}{2}-p\right)} \\
& -i \epsilon\left(x_{1}^{0}-x_{2}^{0}\right) \theta\left(\left(x_{1}-x_{2}\right)^{2}\right) \frac{\Gamma\left(\frac{d-1}{2}\right)}{2 \pi^{\frac{d-1}{2}}} C_{n}^{\frac{d-1}{2}}\left(-x_{1} \cdot x_{2}\right)
\end{aligned}
$$

In odd dimensions the expansion does not exhibit such features as polynomials or logarithms. In all dimensions expansions in terms of the geodesic distance $\sigma$ between $z_{1}$ and $z_{2}$ can be derived by substituting $z=\sin ^{2}(\sigma / 2)$ and reexpanding. This would allow the computation of what is referred to as the expectation value of the renormalized stress-energy tensor, such as those of [7, 23, 24, 25.

While the above construction may be considered satisfactory, one can ask, following the usual way of understanding the massless minimally coupled field [8, 23], whether it would be possible to find a non-anomalous positive quantization on the full test-function space $C_{0}^{\infty}\left(X_{d}\right) \times C_{0}^{\infty}\left(X_{d}\right)$. Of course this quantization would necessarily break the de Sitter symmetry. If it exists, the two-point function 
$W_{n}(x, y)$ solving this problem can be decomposed into the de Sitter invariant part $\widehat{w}_{n}(x, y)$ plus a correction $f_{n}(x, y)$ :

$$
W_{n}(x, y)=\widehat{w}_{n}(x, y)+f_{n}(x, y) .
$$

1) The condition that the theory is canonical, i.e. that $W_{n}(x, y)$ has the right de Sitter invariant commutator $c_{n}(x, y)$, implies that $f_{n}$ must be symmetric:

$$
f_{n}(x, y)=f_{n}(y, x) .
$$

2) The absence of the anomalous term at the RHS of the Klein-Gordon equation satisfied by $W_{n}$ is equivalent to the following non-homogeneous equations for $f_{n}(x, y)$ :

$$
\begin{aligned}
& {\left[\square_{x}-n(n+d-1)\right] f_{n}(x, y)=\left[\square_{y}-n(n+d-1)\right] f_{n}(x, y)} \\
& =-\frac{(-1)^{n+1}(2 n+d-1) \Gamma\left(\frac{d-1}{2}\right)}{4 \pi^{\frac{d+1}{2}}} C_{n}^{\frac{d-1}{2}}(\zeta)
\end{aligned}
$$

note the minus sign at the RHS. The second equation is actually implied by the symmetry of $f_{n}$ and of $C_{n}^{\frac{d-1}{2}}$.

3) $W_{n}(x, y)$ must be positive definite to allow for the quantum mechanical interpretation also of the degrees of freedom that break de Sitter invariance.

4) If satisfied, the condition

$$
\left.f_{n}(x, y)\right|_{\mathcal{E}_{n} \times \mathcal{E}_{n}}=0
$$

guarantees that the breaking of the de Sitter symmetry does not affect the de Sitter invariant physical subspace.

In the massless minimally coupled case a partial solution to this problem satisfying condition 1) 2) and 3 ), but not 4), is exhibited in [8, 23. The explicit time dependence of the two-point function constructed there has been given a physical interpretation in the inflationary context and is regarded as a crucial feature of the model.

In the general case $n>0$, it is easy to find solutions that satisfy either the locality condition 1) or the positive definiteness condition 3), but it does not seem possible to keep both properties (in this connection see also [26]).

In particular the standard KG equation holds by removing $F_{n}^{1}$ and $F_{n}^{2}$ from Eq. (0.14). To restore the positive definiteness one needs to modify the coefficients of the first $n$ terms in the series (0.21). The resulting two-point function satisfies the Klein-Gordon equation and still coincides with $\widehat{w}_{n}$ on $\mathcal{E}_{n} \times \mathcal{E}_{n}$. However it is not canonical and its usefulness outside of the physical subspace appears dubious.

The existence of the above mentioned partial solution to the massless minimal coupled model is due to the fact that this theory is also a limiting case of the positive squared mass theories. However, from the present point of view, the correct massless minimally coupled field is better described by

$$
\left.W_{0}\left(x_{1}, x_{2}\right)\right|_{\mathcal{E}_{0} \times \mathcal{E}_{0}}=\left.\widehat{w}_{0}\left(x_{1} \cdot x_{2}\right)\right|_{\mathcal{E}_{0} \times \mathcal{E}_{0}}
$$

which is a local de Sitter invariant quantization of that field on the space of test functions having zero mean value and is a special instance of our general construction.

We also note that the fields which we have constructed are related to those constructed on the sphere $S_{d}$ (i.e. the "Euclidian" version of $X_{d}$ ) by A. Folacci in [27. However this formalism makes it difficult to study the physical space and the positivity of the metric there. 


\section{References}

[1] G. Feinberg: Possibility of Faster-Than-Light Particles. Phys. Rev. 159: 1089 (1967)

[2] B. Schroer: The quantization of $0>m^{2}$ field equations. Phys. Rev. D 3 (1971) 1764.

[3] O. Nachtmann: Dynamische Stabilität im de-Sitter-raum. Österr. Akad. Wiss., Math.-Naturw. Kl., Abt. II 176:363-379, 1968.

[4] W. Thirring: Quantum field theory in de Sitter space. Acta Physica Austriaca, suppl. IV, 1967, 269.

[5] G. W. Gibbons and S. W. Hawking: Cosmological Event Horizons, Thermodynamics, and Particle Creation. Phys. Rev., D15:2738-2751, 1977.

[6] N. A. Chernikov and E. A. Tagirov: Quantum theory of scalar fields in de Sitter space-time. Annales Poincare. Phys. Theor., A9:109, (1968).

[7] T.S. Bunch, P.C.W. Davies: Proc. R.Soc. Lond. A 360, 117 (1978).

[8] B. Allen: Vacuum States In De Sitter Space. Phys. Rev. D 32 (1985) 3136.

[9] J.Bros, J. P. Gazeau and U. Moschella: Quantum field theory in the de Sitter universe. Phys. Rev. Lett. 73 (1994) 1746.

[10] J. Bros and U. Moschella: Two-point Functions and Quantum Fields in de Sitter Universe. Rev. Math. Phys. 8, 327 (1996) arXiv:gr-qc/9511019.

[11] J. Dixmier: Reprsentations intgrables du groupe de De Sitter Bull. Soc. Math. Fr, 899 (1961).

[12] I.M. Gel'fand and M.I. Graev: Application of the method of horospheres to spectral analysis of functions on real and imaginary Lobachevsky spaces. Trudy Moskov. Mat. Obšč. 11243 (1962).

[13] R. Takahashi: Sur les représentations unitaires des droupes de Lorentz généralisés. Bull. Soc. Math. Fr. 91289 (1963).

[14] I.M. Gel'fand, M.I. Graev and N.Ya. Vilenkin: Generalized functions vol 5 : integral geometry and representation theory Academic Press (1966).

[15] V.F. Molčanov: Harmonic anlysis on a hyperboloid of one sheet. Soviet Math. Dokl. 7 1553, (1966).

[16] J. Faraut : Noyaux sphériques sur un hyperboloïde à une nappe in Lect. Notes in Math. 497, Springer-Verlag, Berlin (1975).

[17] A. M. Polyakov, "De Sitter Space and Eternity," Nucl. Phys. B 797 (2008) 199 arXiv:0709.2899 [hep-th]].

[18] A. M. Polyakov: Decay of Vacuum Energy. arXiv:0912.5503 [hep-th].

[19] J. Bros, H. Epstein and U. Moschella: Lifetime of a massive particle in a de Sitter universe. JCAP 0802:003,2008. Particle decays and stability on the de Sitter universe. e-Print: arXiv:0812.3513 [hep-th].

[20] J. Bros, H. Epstein, M. Gaudin, U. Moschella and V. Pasquier: Triangular invariants, three-point functions and particle stability on the de Sitter universe. Commun. Math. Phys. 295, 261 (2010)

[21] M. Bertola, F. Corbetta and U. Moschella: Massless scalar field in two-dimensional de Sitter universe. Prog. Math. 251, 27 (2007) arXiv:math-ph/0609080.

[22] A. Erdelyi, W. Magnus, F. Oberhettinger, F. G. Tricomi: Higher Transcendental Functions, Vol. 1. New York: McGraw-Hill, 1953 
[23] B. Allen and A. Folacci: The Massless Minimally Coupled Scalar Field In De Sitter Space. Phys. Rev. D 35, 3771 (1987).

[24] D. Bernard and A. Folacci: Hadamard Function, Stress Tensor And De Sitter Space. Phys. Rev. D 34, 2286 (1986).

[25] S. Tadaki: Stress Tensor In De Sitter Space. Prog. Theor. Phys. 80, 654 (1988).

[26] S. P. Miao, N. C. Tsamis and R. P. Woodard: De Sitter Breaking through Infrared Divergences. arXiv:1002.4037 [gr-qc].

[27] A. Folacci: Zero modes, euclideanization and quantization. Phys. Rev. D46, 2553 (1992). 